\title{
A Digital Platform for Marketing Communications in the Mobile and Social Media Space
}

\author{
Otto Petrovic \\ Karl Franzens University, Institute for Information Science and Information Systems, \\ Universitätsstraße 15/G3, 8010 Graz, Austria \\ otto.petrovic@uni-graz.at
}

\begin{abstract}
This paper presents a digital platform for marketing communications developed during the last years. Functionalities of the platform are described according to different categories and by means of case studies together with related results. Also further development strategies are discussed, e.g. the planned enlargement of the platform in the field of social media will include three new modules. A browsing module will enable multidimensional browsing in selected social media. The measurement module will facilitate creation of individual social media portfolios. Finally, the engagement module realizes a uniform user interface between the platform and social media.
\end{abstract}

Keywords: digital marketing platform, mobile advertising, social media marketing, mobile communications.

\section{Introduction}

The digital platform for marketing communications presented in this paper was designed and developed under the guidance of the author in the last four years as well as the strategy for the future development. Compared with other solutions the original contribution of that platform is mainly based on innovations in three areas. Firstly, the presented platform enables an integration of communications via mobile messaging with numerous other digital media, e.g. mobile websites, mobile clients for iPhone and Android, iPad apps, e-mail, RSS feeds and social media. All that channels are used and controlled from the backend functionality described below. Secondly, due to the strong tendency to integrate digital communications with classical media like print, TV, and outdoor advertising, the presented platform supports the linkage between those classical media and new digital media. Thirdly, the platform has an elaborated range of backend functionalities. Those deliver CRM functions for selecting target group together with a wide range of rule based handling of customer interaction.

An important aim of the paper is to present the current development status of the platform by describing the wide range of functionalities in different areas. For each group of functionalities a real life case accomplished by the author is presented together with obtained results. The third aim is to discuss future challenges for 
marketing communications in the digital space and, as a consequence, further development directions of the platform.

\section{Changes in Marketing Communications}

The amount of time that people spend with media has almost tripled within the past four decades and averages ten hours by now [11]. A variety of new media types has evolved simultaneously and communication behavior is becoming more and more inhomogeneous and multifaceted. Two trends are observable in this context. Former key media - TV and radio - are predominately used by older target groups whereas young people turn towards interactive media. Additionally user generated content that is distributed in networks gains significance compared to editorial content that is distributed via broadcasting.

Especially two media gained importance. No communication media was distributed as fast during the past decades and changed communication realities that strong as the mobile phone. Absolute user numbers as well as rate of growth are significantly higher than those of internet. The 4 billion mark of users was passed by the end of 2008 which accounts for about 60 percent of world population [5]. Next to that social media like Facebook, Twitter and Youtube gained importance. Two thirds of all internet users already use social media regularly and time that is spent increases three times as fast as for other internet activities. 93 percent of internet users expect companies to be represented in social media. The analysis of age structure shows that social media is also comprehensively used by senior target groups older than 49 years [10]. Spending for marketing communications align to these new communication realities only step-by-step. United Kingdom was the first big country where internet marketing communications spending for internet with $23,5 \%$ surpassed those for TV accounting for $21,9 \%$ of all spending in the first half of 2009 [13].

In order enable marketing communications to measure up to this changed framework there is need for a digital platform that facilitates management of this constantly increasing complexity. Important tasks in this context are central control of different media, cross-media communication, collecting and using of customer data as well as integration of existing systems such as CRM and ERP. Whereas there already exist commercial systems in the area of classical media and traditional Internet usage, first systems for usage of mobile communication [12] and social media [6,9] are developed in research. Those include hardly any cross-media functionalities and are not evaluated in real usage yet. The purpose of this paper is a contribution to close this gap.

\section{Functionalities of the Platform}

\subsection{Messaging}

The basic form of marketing communications via mobile phone is information broadcast. SMS is qualified to reach almost all users of mobile phones independent from device type. 
Functionalities. In addition to SMS, the platform facilitates further message types: MMS enables transfer of multimedia content and WAP Push is a link that leads to a website which was optimized for display on mobile phones. Another possibility is transmission of emails, which are mobile communication for those recipients who receive emails on their mobile phones. Free choice of message type concerning the single recipient ensures optimum choice of communication form depending on objectives, target groups, and budget.

In order to avoid unwanted messages for the recipient the backend that is explained later offers comprehensive query mechanisms. It is possible to decide who receives certain messages and when. The central messaging administration is also used for quality assurance of messages that are to send.

An additional functionality of the platform is the response option for recipients of the message. It allows the recipient to reply to SMS, MMS and email immediately from the mobile phone. Implementation of mechanisms that allow the sender of the message further processing of responses is especially challenging. Very often only one or two employees are engaged with message transmission and processing of thousands of responses is therefore quite demanding. The platform facilitates automatic conversion of responses into emails and their forwarding according to definable processes.

Even more complex mechanisms of interaction in the area of message transmission are enabled using the mechanism of information and content request by the customer. The customer requests information or other content using a pull mechanism. Certain information or other content is then transmitted using the message types SMS, MMS, email or social media to this customer or to a third party according to the customer data that is stored in the backend and to rules for the processing the dialogue.

Case: Customer Relationship Management of a Retail Bank. The business aims for this retail bank are: low-cost increase of customer contact rate, dedicated services for young target groups and brand image improvement regarding innovation leadership. They decided to use the mobile channel as a fourth channel of communication towards the customer besides over-the-counter business, self service area and telebanking. Requirements were that existing customer data from the CRM system is comprehensively used and that an interface with the core banking system (transaction system) is implemented. This ensures the entire integration of marketing communications via the mobile channel into the bank's other activities. The emphasis was placed on avoidance of spam-messages that could seriously damage the bank's image as a trustworthy partner via the especially sensitive mobile communication channel. Therefore opt-in mechanisms and processes for highly personalized interaction were developed and implemented very carefully. The control cockpit in the backend which is described later takes up a leading role in this context. Essential forms of system usage are: invitations to events, transmission of coupons with the possibility to forward them to friends, personalized information, context-oriented market research, personalized congratulations on birthday, appointment reminder, and incentives for inviting further participants.

A pilot test was conducted in several branch banks in September 2006. The areawide rollout in 380 branches followed in 2009. 150.000 customers were acquired for 
system usage within twelve months showing an upward trend. This value represents a share of $12,5 \%$ of the total population of the target area. One million additional customer contacts could be generated at the same time because of carefully planned communication and interaction activities. The opt-out ration is below three percent. Accompanying market research $(\mathrm{n}=759)$ indicates positive values of system acceptance. $71 \%$ think that the system is either very good or good, $60 \%$ recommended it to someone but only $2 \%$ rated it as bad. Concerning effects on brand image there is a significant improvement in the areas of innovation and customer satisfaction observable. At the same time there was no decrease in trustworthiness which indicates that the especially sensitive mobile channel has been used advisedly.

\subsection{Mobile Market Research}

Market research on the mobile phone offers the essential advantage that a survey can be initiated in a certain context unlike other methods. Examples are surveys concerning customer satisfaction with the check-in service at the airport immediately after checking in or a certain mobile service immediately after using it.

Functionalities. An activity of the participant at the point of sale or usage of a mobile service triggers the transmission of a WAP Push link that is leading to a mobile website. Alternatively an SMS dialogue can be initiated. Then the participant will be able to answer several sets of questions via mobile phone. Different types of questions are available including Likert scale, multiple choice, multiple answer options, numeric questions and yes/no questions. The questions are arranged in blocks that allow adaptive handling depending on answer behavior, using jump orders and filter channels. Direct integration into the backend of the platform enables simplified composition of questionnaires as well as rule-based arrangement and deliver of questions, transmission of immediate feedback, resumption of answering after break-off as well as generating different reports.

Case: Measurement of Perceived Service Quality for a Mobile Network Operator. Main goal of the study [14] was the measurement of customer satisfaction concerning the content download service provided by a mobile network operator immediately after usage. Design of the questionnaire and definition of messaging type for the WAP Push link were processed in the backend. Immediately after the customer's usage of the service the questionnaire is configured according to rules that were defined in the backend of the platform then personalized and transmitted adapted to mobile phone type using the rendering engine in an optimized way. The response rate of this mobile survey was $6,4 \%$ which is four times higher than the response rate of the online survey $(1,7 \%)$ that was conducted for reasons of comparison. $53 \%$ of the participants who finished the WAP survey within seven days actually completed it within one hour after start in contract to the online survey where only $27 \%$ of the participants finished the survey within the first day. These facts indicate that an addressee of a mobile survey decides to take part either immediately after receiving the invitation link or not at all. 


\subsection{Interactivation of Traditional Media}

Traditional media of marketing communications such as newspaper, advertisement, TV spots, or billboards basically follow a broadcast approach. A message is distributed to as many members of the target group as possible. Interaction is not intended or only indirectly possible like in form of letters to the editor or calls by viewers or listeners. Interactivation of traditional media enables utilization of the mobile phone as an immediate feedback channel for marketing communications in traditional media and hence turns them into interactive media.

Functionalities. In a first step the company creates a physical medium for their advertising message. This could be a newspaper advertisement, billboards, a TV spot or an object like a t-shirt or the wall of a building. After that, an interface with the mobile phone is implemented on this medium. The platform supports SMS numbers, 2D-codes [7], Bluetooth [8], and Near Field Communication (NFC) [4]. Depending on type and configuration of the implemented interface the customer receives a call for interaction when coming closer to the vehicle of the advertising message (Pushtechnology) or can initiate interaction himself (Pull-technology). Access of a mobile website, content download and SMS dialogue are supported forms of interaction. The profound integration into the backend of the platform allows definition of detailed rules for starting and processing the interaction as well as generation of comprehensive reports concerning customer behavior.

Case: Interactivation of Billboards using 2D Codes and Bluetooth. A billboard campaign was interactivated for the exhibition of a 25.000 year-old statue in a museum of historical art using this module of the platform. Main purposes were increasing attention to the billboard, invite for interaction and stimulating viral effects. The billboards were therefore equipped with 2D codes. Activation of an applicable reader on the mobile phone enabled passers-by to download a game that was especially created for mobile phones including information concerning the statue. They could also forward the game to friends and acquaintances immediately from their mobile devices. Additionally a Bluetooth interface was implemented in order to call attention of passers-by with enabled Bluetooth recognition to the possibility to get further information concerning the statue on their mobile phones. Within three weeks 3.200 mobile phones with enabled Bluetooth recognition were detected. Invitation for download was accepted 630 times which represents a response ratio of $20 \%$.

\subsection{Mobile Websites and Mobile Apps}

The increasing penetration with smart phones and their ability to display mobile websites and execute special application on the device fosters demand for mobile websites and mobile apps [3]. This effect is enforced by low-cost data tariffs provided by mobile network operators often in form of flat fees. This is why mobile websites and mobile apps increasingly replace SMS-based forms of marketing communications [2].

Functionalities. In contrary to usage of websites via PC there is no uniform standard for their usage on mobile phones. Devices differ a lot concerning data formats for pictures and videos that are supported, display size, screen resolution and browser functionalities. For this concern a Rendering Engine was implemented into the 
platform which recognizes the type of mobile device and enables optimized delivery of the website. At the moment the Rendering Engine includes 5.000 different types of mobile devices while this base is continuously updated and enlarged. It enables output of the website at the best concerning markup language, resolution, display size and data format. Data from websites gets into the Rendering Engine either via a dedicated mobile website, via integration of existing data using an RSS feed or via direct integration of the Rendering Engine into the particular Content Management System (CMS).

Because of increasing processing power of smart phones the number of mobile apps that are developed and used also increases. These apps enable usage of a particular mobile device's functionalities as well as offline usage of applications and data. Moreover the user perceives an emotional quality of actually "possessing" something which positively affects willingness to pay for it. This assumption is supported by enormous sales volumes e.g. for Apple AppStore where more than one billion apps were downloaded within nine month after opening [1]. The plattform is able to deliver messages, information and coupons into a mobile application based on rules for every single user of a certain application. Further functionalities of the platform are access to customer data, triggering of interaction via other channels, direction of interaction and content of the app as well as a comprehensive reporting.

Case: Launch of a New Car Model and Development of a Mobile Loyalty Card. The launch of a new car model was supported by a mobile website. This website could be accessed either via directly inserting the URL or via 2D codes in newspaper advertisings. Next to current news concerning the model this website included a comprehensive description of the new model as well as of alternative models, the range of products and the possibility to contact a nearby merchant in order to make an appointment for a test drive. In the case of GPS enabled devices the nearby merchant was immediately suggested. A comprehensive reporting system between merchants and automotive manufacturer was implemented in order to centrally collect and analyze the customer data generated subsequent to test drives. The mobile website had 2.500 visits within 30 days and 8.000 page views. $90 \%$ of the visitors accessed the mobile website using the $2 \mathrm{D}$ codes integrated in newspaper advertising. The most visited areas were those concerning the newly launched car model.

Additionally a mobile app in form of a mobile loyalty card was developed integrated in the platform. Depending on the stored interaction mechanics the backend enables transmission of personalized content to each single customer according to deposit on the loyalty card. It is also possible to transfer personalized coupons to the app. Clicking a certain coupon initiates display of a customary EAN code that can be read by standard scanners on the point-of-sale.

\section{The Backend}

\subsection{Functionalities of the Backend}

An essential functionality of the backend is the control cockpit. Communication Planning enables selection of customers with whom a certain form of communication is to be initiated. Next to demographic criteria drawn from customer master data also 
behavior-oriented data and transaction data is used. For instance certain messages are only sent to those customers that are younger than 20 years and who did not buy a product within the past two months. The functionalities concerning content selection support personalization of content like personalized address on the one hand and on the other hand quality assurance of sent content in decentralized organizations. For example it is possible that the communication department defines various standard contents like birthday messages, messages in case of expiration of a contract or a message addressing negative news coverage concerning the company. The particular departments or branches can then use these drafts for sending messages to their customers. At the same time the module for communication planning displays costs related to the particular selection of target groups and content like for outgoing SMS.

The Reporting Module conduces to analysis of customer behavior. Examples are response ratios for certain transmitted messages or surveys, redemption rates of coupons, access numbers of mobile websites or forwarding rate of viral content. All reports can be displayed either on mobile devices or in the web. The reports are supplemented by a thorough monitoring of system performance. A further module is dedicated to User Administration. It includes definition of groups, roles and authorization in order to define who is allowed to administer which system transactions. Campaign and Program Control is used for grouping if single activities that are topically related. It is possible to define rules for editing customer feedback concerning sent messages and also to decide on certain types of content according to customer behavior. All these functionalities are at the disposal of all communication channels illustrated above. Integration possibility of the backend into existing IT-systems is of paramount importance in order to avoid isolated solutions. Fig. 1 shows the integration of the platform into existing ERP- and CRM-systems, transaction systems at the point-of-sale, call center activities and campaign management software.

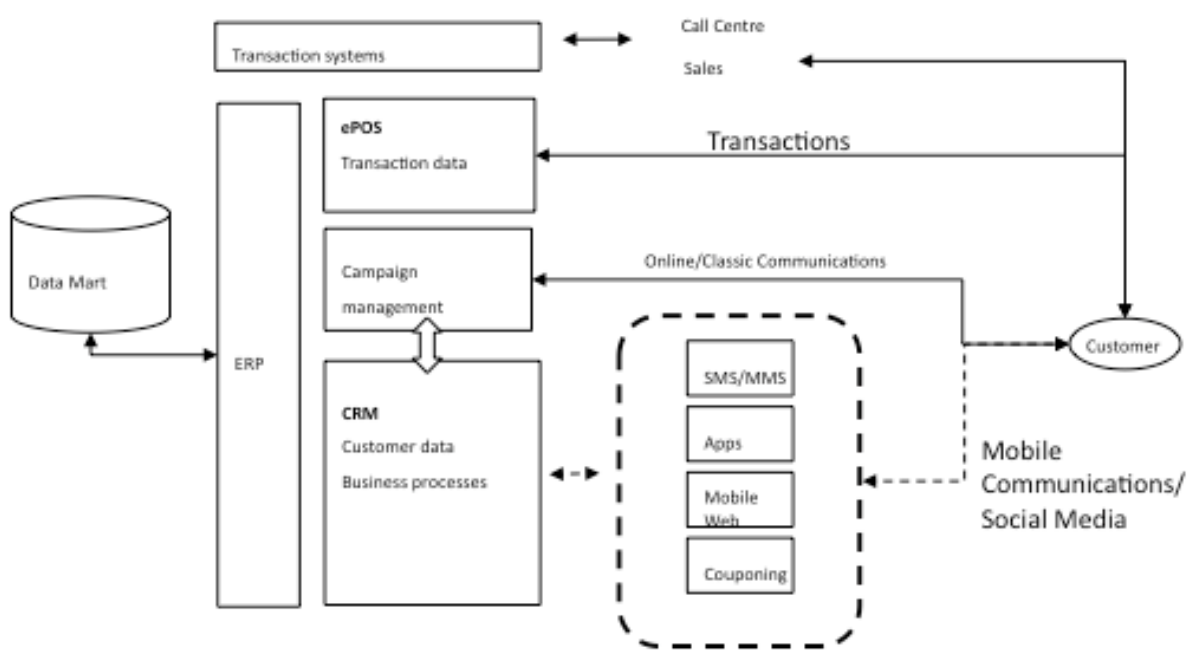

Fig. 1. Integration of the platform in other IT-Systems 


\subsection{Technical Implementation of the Backend}

The technical implementation is done in Java as far as possible and reasonable. For interactions that are adaptable during runtime Groovy, which is based on JDK (Java Development Kit) and therefore enables integration into the platform, is used as script language. The server system is based on the specifications of the Java platform Enterprise Edition. This covers following aspects of platform software: webcontainer-infrastructure, administration of transactions and relational data base access. Integrating platform (middleware): Apache Tomcat. Data base: In order to allow secure and scalable administration of a huge amount of users MySQL (data base engine: InnoDB) is used. Web-applications-framework: Struts-framework to ensure a uniform user interface with a MVC-structure (model-view-controller). Objectrelational mapping: Data base tables are configured by means of Hibernate-library and implemented in Java-object structure. Therefore business logics and actual data storage are uncoupled in the coded areas of the platform. Reporting-tool: Reports are generated using the reporting tool BIRT. Views are defined that allow uncoupling of report queries and actual table structure.

\section{Future Development}

\subsection{From Messaging to Cross-Media Communications and Dedicated Adservices}

One important factor for further development of the platform is the advent of smart phones together with mobile broadband connectivity and flat fees for using the internet. That bundle of innovation leads to a relative gain of mobile internet-based communications in form of mobile websites, mobile clients and social media via traditional short message services. The main challenge is to enable consistent brand strategies and consumer interaction mechanisms independent from differing digital media used by the target group. The most important challenge is not only to support the communication of a company with its clients but also to handle all the responses as well as to influence the communication among brand relevant peer groups in social media. Additionally upcoming dedicated ad-services like Google's AdMobs or Apple's iAd will challenge the interface of the current platform. Monitoring Systems like flurry.com deliver completely new consumer insights in the field of application usage and thus new communication opportunities. But the most important challenge will come from social media.

\subsection{Future Deployment of Social Media}

Marketing communications gained a totally new dimension caused by social media (e.g. Facebook, Twitter, Blogs, Wikis, and further more). It is not about developing messages and then penetrating the market any more but about engaging in existing, hard to control communication processes of (potential) customers and stakeholders that are relevant to the brand. In order to use these new possibilities the platform is currently enlarged by three additional modules. 
Browsing Module: This module includes a freely configurable dashboard that enables multidimensional browsing in social media of one's own choice. Dimensions can be for instance brand values. The Browsing Module displays relevant activities in social media (postings, uploaded videos and photos) multidimensional on the screen in realtime. The creative department can now browse through this space and get an emotionally loaded picture of the activities that are of high relevance to his own or his client's brand values.

Measurement Module: This module facilitates creation of own portfolios of relevant social media. The Social Media Marketing Engine will then automatically access transaction data from the chosen websites which replaces manual research. Certain key data are listed for each social medium (e.g. number of followers, tweets, friends, watchers, stickiness, time on site). This functionality causes identification of those social media that are of high importance to the enterprise as well as of opinion leading users and content that is especially influential and often virally passed on.

Engagement Module: By means of a uniform user interface it is possible to communicate within the sites that are listed in the Social Media Portfolio. Various activities in social media (search, upload, matching of posts and profiles, tagging, voting, categorization, administration of authorization) can be administered by using APIs that are provided by the particular operator. The module particularly supports the following forms of communication design in social media: direct dialogue with customers, distribution of viral content via seeding (spreading of media content targeted especially at opinion leaders), creation of a mass appeal by brand owners, complaint management.

\subsection{Technological Implementation of the Integration of Social Media}

The interfaces between the platform and external systems will be adapted to and enlarged according to APIs that are used in leading social networks. Main purpose is to create a central interface between the platform and the networks and then use it for all functionalities described above. Following APIs will be implemented: Twitter: usage of simple http accesses via GET/POST. It is necessary to implement REST API to enable access to core data as well as access via Streaming API. Processing of the responses is conducted in XML/JSON respectively via http status codes for malfunction reports. Usage of existing Open Source Java Libraries (Twitter4J, Java-twitter, jwitter) is planned. Facebook: Facebook Connect is used as the central API in order to access all functionalities. Existing JavaScript SDK is used for the development of the frontend. Usage of existing Open Source Java Libraries (Facebook-Java-API) is planned. Youtube: Different APIs are available enabling data access and integration of players. The focus is on Data API that offers access to manifold functionalities. Data API uses http accesses as well as XML and additionally Java Client Libraries can be used. Fig. 2 shows the technical implementation of the platform enlarged by integration of social media. 


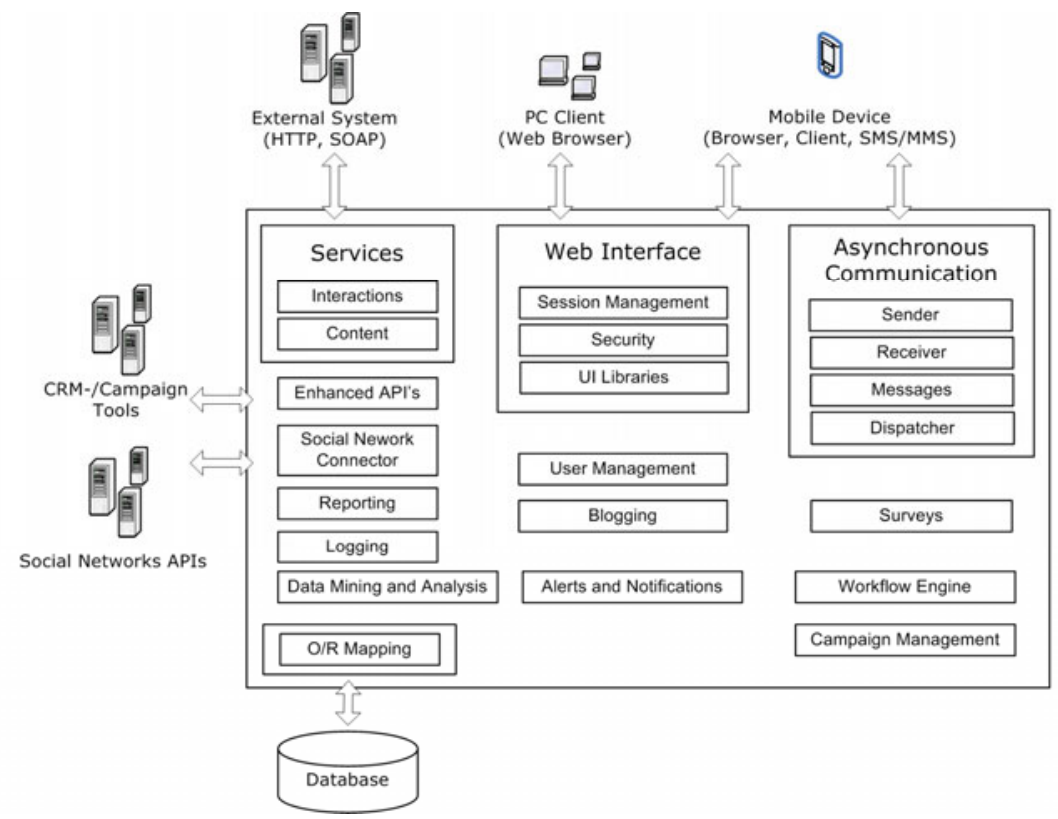

Fig. 2. Technical implementation of the platform enlarged by integration of social media

Acknowledgments. The developer teams of the evolaris foundation and of scoop and spoon did the programming of the platform. The team of scoop and spoon will code the future development. All that work is strongly appreciated.

\section{References}

1. Apple, http: / /www .apple.com/at/itunes/billion-app-countdown/ (accessed 05.05.2010)

2. Little, A.D.: Exane BNP Paribas: Mobile Internet: blessing or curse? 9th edn. (2010)

3. Businesswire: GetJar Reveals That Mobile Apps Will Outsell CDs (2012), http: / / www.businesswire.com/portal/site/home/permalink/ ?ndmViewId=news_view\&news Id=20100316007140\&newsLang=en (accessed 05.05.2010)

4. Finkenzeller, K., Kenneth, C.: RFID Handbook: Fundamentals and Applications in Contactless Smart Cards. In: Identification and NFC. Wiley, UK (2010)

5. International Telecommunication Union: Mobile Phone Subscribers Pass 4 Billion Mark, http: //www.itu.int/ITU-D/ict/newslog/Mobile+Phone+ Subscribers+Pass+4+Billion+Mark . aspx (accessed 18.05.2009)

6. Jansen, B.J., Sobel, K., Cook, G.: Gen X and Ys attitudes on using social media platforms for opinion sharing. In: Proceedings of the 28th International Conference on Human Factors in Computing Systems, pp. 3853-3858. ACM, New York (2010)

7. Kato, H., Tan, K.T., Chai, D.: Barcodes for Mobile devices. Cambridge University Press, Cambridge (2010) 
8. Maron, M., Magnus, S., Read, K.: An Empirical Study to Evaluate the Location of Advertisement Panels by Using a Mobile Marketing Tool. In: Proceedings of the 2009 Eigth International Conference on Mobile Business, pp. 196-202. IEEE Computer Society, Washington (2009)

9. Morris, M.R., Teevan, J., Panovich, K.: What Do People Ask Their Social Networks, and Why? A Survey Study of Status Messages Q\&A Behaviour. In: Proceedings of the 28th International Conference on Human Factors in Computing Systems, pp. 1739-1748. ACM, New York (2010)

10. Nielsen: Global Faces and Network Places: A Nielsen Report on Social Networking's New Global Footprint, http://blog.nielsen.com/nielsenwire/wp-content/ uploads / $2009 / 03 /$ nielsen_globalfaces_mar09.pdf (accessed 05.05.2010)

11. Reitze, H., Ridder, C.: Massenkommunikation VII. Eine Langzeitstudie zur Mediennutzung und Medienbewertung 1964-2005. (Mass communications 7. A long-term study of media usage and media appraisal 1964-2005.) Nomos, Baden-Baden (2006)

12. Richardson, I., Third, A.: Moblogging and belonging: new mobile phone practices and young people's sense of social inclusion. In: Proceedings of the 2nd International Conference on Digital Interactive Media in Entertainment and Arts, pp. 73-78. ACM, New York (2007)

13. Sweney, M.: Internet overtakes television to become biggest advertising sector in the UK, The Guardian (Wednesday September 30, 2009), http: / /www.guardian.co.uk/media/2009/sep/30/ internet-biggest-uk-advertising-sector (accessed 05.05.2010)

14. Wallisch, A., Maxl, E.: A customer satisfaction study for Vodafone live. In: Maxl, E., Döring, N., Wallisch, A. (eds.) Mobile Market Research. Neue Schriften zur OnlineForschung 7 (New papers concerning online research 7), pp. 276-283. Herbert von Halem Verlag, Cologne (2009) 